\title{
La sensibilisation à la pharmacie : une action de tous les jours
}

\author{
par Bruce Millin
}

$\int \mathrm{e}$ e rédige ce commentaire tout juste avant le début d'un nouveau Mois de sensibilisation à la pharmacie. Cette campagne constitue une excellente occasion de mieux faire connaître l'influence de la pharmacie sur les patients. Elle offre aussi aux organisations et aux praticiens la chance de souligner le fait que le pharmacien est l'expert des médicaments du système de santé. Cette année, la campagne vient à point nommé en raison de la récente couverture médiatique (déclenchée par un reportage d'enquête de l'émission Marketplace de la CBC) remettant en question la pratique de la pharmacie et les soins qu'elle offre. Bien que le reportage de Marketplace n'ait pas été centré sur la pratique de la pharmacie dans un établissement de santé, il a néanmoins terni l'image des pharmaciens ainsi que leur rôle essentiel qui est d'assurer la sécurité et l'efficacité de la pharmacothérapie offerte aux patients. Il serait tentant de rejeter le reportage sous prétexte qu'il ne s'applique pas à la pharmacie hospitalière ou de rester sur la défensive devant ses conclusions et de souligner les failles de l'enquête. Mais, je crois qu'il ne faut pas perdre de vue ce dont parle le reportage et qu'il faut le voir comme une occasion pour souligner les systèmes et les automatismes régulateurs en place visant à déceler, résoudre et prévenir les problèmes pharmacothérapeutiques.

Si le Mois de la sensibilisation à la pharmacie offre des occasions de mieux faire connaître le rôle des pharmaciens, il ne faut pas oublier que la promotion de notre profession est une action de tous les jours. Ainsi, en 2015 et pour les années à venir, la Société canadienne des pharmaciens d'hôpitaux (SCPH) continuera à exercer son leadership pour ce qui est de participer à la sécurité et à la qualité des soins accordés aux patients. Des exemples phares de ce travail sont les stratégies pour l'excellence en pratique, notamment le projet SCPH 2015, la promotion de la trousse à outils pour la valorisation conçue par la SCPH ainsi que les collaborations en cours avec diverses organisations, notamment : Santé Canada, l'Association nationale des organismes de réglementation de la pharmacie, l'Association des pharmaciens du Canada, l'Association canadienne de pharmacie en oncologie, l'Association des pharmaciens des établissements de santé du Québec, l'Institut canadien pour la sécurité des patients, l'Institut pour la sécurité des médicaments aux patients du Canada et l'Association canadienne des techniciens pharmaceutiques.

Le travail de valorisation de la SCPH, tant au niveau national qu'au niveau des sections, est nécessaire et souhaitable, mais les efforts de valorisation par les praticiens eux-mêmes sont cruciaux. Postés en première ligne, devant effectuer leur travail quotidien et répondre aux exigences des soins aux patients, les praticiens n'arrivent pas toujours à voir comment, en tant qu'individus, ils peuvent promouvoir le changement et valoriser la profession. Je vous invite tous et toutes à envisager chacun des échanges qu'un pharmacien a avec un professionnel de la santé ou un patient comme un prétexte de valorisation, non seulement de la profession, mais aussi des intérêts des patients. Voilà une formule gagnante.

[Traduction par l'éditeur]

Bruce Millin, B. Sc. (Pharm.), ACPR, est président et agent de liaison interne pour la Société canadienne des pharmaciens d'hôpitaux. 